\title{
Temperature dependence of interfacial properties and viscosity of nanofluids for droplet-based microfluidics
}

\author{
S M Sohel Murshed, Say-Hwa Tan and Nam-Trung Nguyen* \\ School of Mechanical and Aerospace Engineering, \\ Nanyang Technological University,Singapore 639798, Republic of Singapore \\ *Email: mntnguyen@ntu.edu.sg
}

\begin{abstract}
Interfacial tension and viscosity of a liquid play an important role in microfluidic systems. In this study, temperature dependence of surface tension, interfacial tension and viscosity of a nanofluid are investigated for its applicability in droplet-based microfluidics. Experimental results show that nanofluid having $\mathrm{TiO}_{2}$ nanoparticles of $15 \mathrm{~nm}$ diameters in deionized water exhibit substantially smaller surface tension and oil-based interfacial tension than those of the base fluid (i.e. deionized water). These surface and interfacial tensions of this nanofluid were found to decrease almost linearly with increasing temperature. The Brownian motion of nanoparticles in the base fluid was identified as a possible mechanism for reduced surface and interfacial tensions of the nanofluid. The measured effective viscosity of nanofluid was found to be insignificantly higher than that of base fluid and to decreases with increasing fluid temperature. The dependency on temperature of the droplet formation at T-junction of a microfluidic device is also studied and nanofluid shows larger droplet size compared to its base fluid.
\end{abstract}

\section{Introduction}

Nanofluid, a new and innovative class of composite fluid, is engineered by dispersing nanometer-sized particles in conventional fluids such as water. From the investigations in the past decade, nanofluids were found to exhibit higher thermophysical properties such as thermal conductivity, thermal diffusivity and viscosity than those of base fluids thermal diffusivity and viscosity than those base fluids[1-5]. The potential applications of nanofluids include microelectronics, microfluidics and transportation [6]. There are mainly two technique for synthesizing nanofluids, which are the two-step process and the direct evaporation technique or the single-step process[6].In the two-step process, dry nanoparticles are first produced by an inert gas condensation method and they are dispersed into a fluid. The direct evaporation technique synthesizes nanoparticles and disperses them into a fluid single step. However, most researchers use the two-step process to produce nanofluids by dispersing commercial or self-produced nanoparticles in a liquid.

With increased interest in microfluidic systems, interfacial phenomena are attracting more attention from researchers worldwide. In particularly, interfacial tension and viscosity of liquid play an important role in continuous flow- and droplet-based microfluidics. For instance, in droplet-based microfluidics, the size of droplet can be controlled with the interfacial tension and dynamic viscosity, which are directly 
related to the droplet size at a fixed shear condition. Besides microfluidics, many industrial applications such as spreading of droplet, surface acting agent and many chemical engineering applications rely on the precise control of the surface tension. The temperature dependence of interfacial tension and viscosity of the liquid is also crucial in thermally mediated droplet formation [7,8] and droplet breakup [9]. In the field of nanofluids, only Wasan and Nikolov [10] studied the spreading of nanofluids on solid surface and found that the existence of nanoparticles near the liquid/solid contact line can improve its spreading. To the best of our knowledge, no investigation was reported on the characterization of temperature dependence of surface and interfacial tension of nanofluids.

Although viscosity influences the interfacial phenomena and flow characteristics, very few studies have been performed on the effective viscosity of nanofluids as a function of volumetric loading of nanoparticle [11-13]. The primary results from these studies indicate that the viscosity of a nanofluid increases with increasing nanoparticle volume fraction. In addition to the very few experimental studies, no theoretical model is available for the prediction of effective viscosity of nanofluids as a function of temperature and particle volume fraction. It is worthwhile to study temperature-dependent viscosity of nanofluids for their potential applications especially in microfluidic systems.

Even though droplet-based microfluidics is an emerging research area and is attracting much attention from the microfluidics research community [14-17], no study has been reported in the literature on the nanofluid's droplet formation and manipulation. Aiming at the potential applications of nanofluids in droplet-based microfluidics, we investigate the temperature dependence of the surface tension, the interfacial tension with mineral oil, and the viscosity a nanofluid. Results on temperature dependence of droplet formation at T-junction of a microfluidic device are also presented and discussed.

\section{Theoretical}

The surface tension of a liquid and interfacial tension between two immiscible liquids can easily and precisely measured but cannot directly be determined due to the unknown empirical parameter in the existing models such as the Langmuir model [18]. Therefore, experimentally fitted correlations are widely used to estimate the surface and interfacial tensions. Moreover, no theoretical study has been reported on these interfacial phenomena and temperature-dependent viscosity of nanofluids. In this study, two empirical expressions are used to estimate the surface and interfacial tensions of sample fluids.

The surface tension $\left(\mathrm{Nm}^{-1}\right)$ of water, which is most commonly used in microfluidics can be estimated from [19]

$$
\eta_{\mathrm{g}}=\left(0.076-0.00017 \mathrm{~T} \mathrm{~K}^{-1}\right),
$$

where $T$ is the temperature of the system and $\gamma_{\mathrm{lg}}\left(\mathrm{Nm}^{-1}\right)$ is the surface tension of liquid in the gaseous phase. This experimentally fitted expression is only related to temperature. However, the surface tension of a liquid also strongly depends on the 
presence of contaminants (e.g. dirt) or dispersion agents such as surfactants.

Based on the surface tension of each of a liquid/liquid system, Girifalco and Good [20] developed a model to estimate the interfacial tension, which is given as:

$$
\gamma_{\mathrm{ab}}=\gamma_{\mathrm{a}}+\gamma_{\mathrm{b}}-2 \Phi \sqrt{\gamma_{\mathrm{a}} \gamma_{\mathrm{b}}}
$$

where $\gamma_{a}$ and $\gamma_{b}$ are the surface tensions of phase a and $\mathrm{b}$, respectively and $\Phi$ is a constant which is equal to the ratio of energies of adhesion and cohesion for two phases. The values of $\Phi$ for a number of different liquid/liquid systems are given by Girifalco and Good [20].

The effective viscosity ( $\eta_{\text {eff }}$ ) of dilute suspensions is commonly determined by Einstein's viscosity model [21] given by

$$
\eta_{\mathrm{eff}}=\eta_{\mathrm{f}}(1+2.5 \phi)
$$

From equation (3), it can be seen that this classical model depends on particle volume fraction $(\phi)$ and base fluid viscosity $\left(\eta_{f}\right)$. Equation (3) can also be used to estimate the effective viscosity of nanofluid at various temperature provided that the vicosity of the base fluid at the corresponding temperature is known.

\section{Experimental}

\subsection{Sample preparation}

Nanofluid was prepared by dispersing 0.1 vol\% of spherical shaped titanium dioxide $\left(\mathrm{TiO}_{2}\right)$ nanoparticles of $15 \mathrm{~nm}$ diameter in deionized water (DIW). To ensure proper dispersion of nanoparticles the sample nanofluid was homogenized by using an ultrasonic dismembrator (Fisher Scientific Model 500) and a magnetic stirrer. Mineral oil (Sigma 5904, Sigma-Aldrich, Singapore) was used as other liquid for interfacial tension measurement. While mineral oil with $2 \%$ w/w Span 80 surfactant (Sigma S6760, Sigma-Aldrich, Singapore) was used as the carrier fluid in microchannel, deionized water and nanofluid with $0.05 \%$ w/w fluorescence dye (Sigma F6377, Sigma-Aldrich, Singapore) were used as the aqueous fluids for the droplet formation. The viscosity of the mineral oil with surfactant is $23.8 \mathrm{mPa}$ s and its temperature dependence was characterized previously in [1].

\subsection{Measurements}

A Surface Tensiometer (DCAT 21, Data Physics Instruments GmbH, Germany) was used to measure the surface tension of sample fluids at different temperatures ranging from $25-55^{\circ} \mathrm{C}$. The interfacial tension of the sample fluids was measured with a video system (FTA 200, First Ten Angstroms, Taiwan). Since this system (FTA 200) does not have any facility to measure the interfacial tension as a function of temperature, the mineral oil (Sigma 5904, Sigma-Aldrich, Singapore) was heated in a small container made of Pyrex glass to obtain the different temperatures. Viscosities of deionized water and sample nanofluid were measured with a Lowshear (LS 40, Mettler Toledo, Switzerland) Rheometer at different temperatures. The 
experimental facilities were calibrated by measuring the properties of known fluid i.e. deionized water. Based on the deviation between the standard and the measured values of the propertied of deionized water at room temperature, the present measurement error was estimated to be within 1\%. All measurements were performed at atmospheric pressure.

Droplets in microchannels are usually generated by two configurations, which are T-junction and flow focusing through a small orifice. In this study, the temperature dependence of droplet formation and size manipulation is investigated at $\mathrm{T}$-junction of microfluidics device. The device was fabricated using micromachining of glass and polydimethylsiloxane (PDMS). The temperature sensor and microheater, which were made of thin-film platinum, were patterned using photolithography and lift-off technique. While the sensor is used to detect the temperature at the microchannels, the microheater is to provide localized heating. Titanium was used as the adhesion layer between glass and platinum. The microchannel network was fabricated in PDMS using soft lithography. The master mold was fabricated by photolithography of the thick-film resist SU-8 using a transparency mask. The glass wafer with the patterned microheater and microsensor was subsequently coated with a thin PDMS layer before being bonded to the PDMS part containing the microfluidic network. This step makes sure that all channel walls have the same properties. Bonding is achieved using oxygen plasma treatment on both PDMS surfaces. The widths of the carrier channel and the injection chanel are $150 \mu \mathrm{m}$ and $50 \mu \mathrm{m}$, respectively. All channels are $90 \mu \mathrm{m}$ deep. The temperature sensor was calibrated so that its resistance values can be used for in situ temperature measurement. Schematic of the T-junction is shown in figure1.

Two precision syringe pumps (KD Scientific Inc., USA) were used to drive the oil and the aqueous fluid. The temperature sensor was calibrated before the experiments. The temperature, which was adjusted by changing the voltage of the heater, was monitored through the resistance of the temperature sensor. An epifluorescent inverted microscope with a filter set (Nikon B-2A, excitation filter for $450-490 \mathrm{~nm}$, dichroic mirror for $505 \mathrm{~nm}$, and emission filter for $520 \mathrm{~nm}$ ) was used to observe the droplets. A sensitive interline transfer CCD camera (HiSense MKII, Dantec Dynamics, Denmark) was employed for recording the droplet images which were then processed by a customized MATLAB program for determining the droplet diameter.

\section{Results and discussion}

\subsection{Temperature dependence of surface and interfacial tensions}

Figure 2 depicts the comparison of measured temperature-dependent surface tensions of sample fluids (i.e. deionized water and nanofluids) and the predictions by equation (1) as well as data obtained from the literature for water [22]. It can be seen that dispersion of such a small volume fraction i.e. 0.1 volume $\%$ of $\mathrm{TiO}_{2}$ nanoparticles considerably reduces the surface tension of deionized water, which was found to decrease almost linearly with increasing temperature (figure 2). It is not clear about the observed trend of surface tension of deionized water, which differs with the literature data [22] at temperature beyond $25^{\circ} \mathrm{C}$. Besides deionized water in this study and normal tap water in the literature data[22], there could be some other reasons 
related to the measurement technique and equipments for the observed differences (figure 2).

Figure 3 shows that nanofluid exhibits significantly smaller interfacial tension in mineral oil compared with that of base fluid (DIW). The measured results are in good agreement with the calculated results from the classical Girifalco and Good model given by equation (2). It is noted that the measured value of surface tension of each phase (DIW, nanofluid and oil) was used to estimate the interfacial tension by this model. In contrast to deionized water, the measured data for interfacial tension of nanofluid show a nearly linear trend of decreasing with increasing temperature (figure 3). Since the values of $\Phi$ for DIW/mineral oil- and nanofluid/mineral oilbased systems are not available in the literature, they are chosen to be 0.54 and 0.67 , respectively to fit the experimental results. Nevertheless, these fitted values are within the range of given values for water in similar organic liquids [20]. Interestingly, the present experimental results introduced two new values of $\Phi$ (i.e. 0.54 and 0.67 ) to be used in Girifalco and Good model for estimating the interfacial tensions of deionized water/oil and nanofluid/oil-based systems.

The results presented in figures 2 and 3 clearly demonstrate that the surface and interfacial tensions of this nanofluid are significantly smaller than those of the base fluid. The reason is that nanoparticles can easily experience Brownian motion and interact with the liquid molecules as well as the nanoparticles themselves resulting in a reduced cohesive energy at the interface. Brownian motion is also efficient in redistributing the particles to the lowest total free energy at the interfaces[23]. Moreover, an elevated temperature intensifies the Brownian motion and it is known that the lower the cohesive energy the smaller the surface or interfacial tension. Futhermore, nanoparticles can be adsorbed at the interfaces (i.e. liquid-air or liquidliquid) and function in similar ways to surfactants to reduce the surface tension or interfacial tension $[23,24]$. Similar to the alteration of thermophysical properties $[4,12,13]$, the addition of a small amount of nanoparticles into liquid can change its surface properties such as surface and interfacial tensions.

\subsection{Temperature dependence of viscosity}

Experimental results on temperature-dependent viscosity of deionized water, nanofluid and standard data of water [25], results from the literature [26] as well as the calculated data from Einstein's model[21] are shown in figure 4. As can be seen from figure 4 , the calculated data (using the measured viscosity data of DI water ) from Einstein's model are almost the same as those of DI water and this classical model significantly underestimates the effective viscosity of the nanofluid. The measured effective viscosities of sample fluids are found to decrease significantly with increasing fluid temperature (figure 4). However, compared with base fluid, no significant change in viscosity of nanofluid was observed. The slightly higher viscosity of nanofluid could be due to particle clustering, interactions and interparticle potential such as van der Waals force.

\subsection{Temperature-dependent droplet formation at T-junction}

As the droplet formation process at $\mathrm{T}$-junction is adequately discussed in the literature $[15,27,28]$, it will not be elaborated here. We will however identify the 
regime of droplet formation in our experiments. In this study, the flow rates of the carrier fluid (oil) and the aqueous fluid (DI water and nanofluid) were kept at constant values of $300 \mu \mathrm{lh}^{-1}$ and $60 \mu \mathrm{lh}^{-1}$, respectively. A recorded image of the droplet formation process at a microfluidic $\mathrm{T}$-junction is shown in figure 5 . The time scale of a droplet formation at the T-junction was within $500-700 \mathrm{~ms}$ under the aforementioned flow rates of the carrier fluid and the aqueous fluids.

In microfluidic droplet formation, the dimensionless capillary number $\mathrm{Ca}$ is the most important parameter characterizing the droplet formation regime through the relative importance of viscous stress and interfacial tension or capillary pressure. This capillary number is defined as $C a=\eta_{c} u_{c} / \gamma$, where $\eta_{c}$ and $u_{c}$ are the viscosity and flow velocity of the carrier fluid, respectively and $\gamma$ is the interfacial tension of the carrier fluid/aqueous fluid. The flow velocity of the carrier fluid i.e. oil is determined from $u=(Q / h w)\left(6.17 \mathrm{mms}^{-1}\right)$, where $Q$ is the flow rate, $h$ and $w$ are the depth and width of the channel, respectively. Using this flow velocity and known values of viscosity and interfacial tension of carrier fluid, the capillary number $(\mathrm{Ca})$ at room temperature for deionized water and nanofluid were calculated as $2.8 \times 10^{-3}$ and $4.1 \times 10^{-3}$, respectively. According to Garstecki et al. [27] for such small values of capillary numbers, the droplet formation is in the squeezing (pressure-dominated) regime suggesting that interfacial tension dominates viscous stress.

Droplet formation of both DI water and nanofluid is characterized at different temperatures. Figure 6 shows temperature dependence of droplet size in a T-junction microfluidic device. It can be seen that the droplets size of nanofluids are larger than its base fluid and the dependency on temperature is more significant compared with the base fluid. Since the effective viscosity of nanofluid does not change significantly with temperature (figure 4), the formation of droplet is governed by the interfacial tension, which is much smaller (figure 3) for nanofluid than that of deionzed water. At this stage, it is however, not clear how the nanoparticles can contribute to increasing the droplet size apart from the interfacial tension.

\section{Conclusions}

Nanofluid was found to exhibit considerably smaller surface tension and oil-based interfacial tension than those of the base fluid itself. Experimental results also demonstrate that surface and interfacial tension of nanofluid decrease with increasing temperature. The Brownian motion and adsorption of the interfaces were identified as mechanism for reduced surface and interfacial tensions of nanofluid. Compared to base fluid (i.e. deionized water) no significant change in viscosity of nanofluid was found in this study. The dispersion of small volume fraction of nanoparticles significantly increases the droplet size of deionized water formed at a microfluidics Tjunction and the droplet size was found to increase nonlinearly with increasing temperature. Nevertheless, these results show the applicability of nanofluids in droplet-based microfluidics. It is also imperative to conduct more studies in order to elucidate the mechanisms for temperature-dependent interfacial properties, viscosity, and microdroplet formation of nanofluids. 


\section{Acknowledgements}

The authors acknowledge the support from the Agency of Science, Technology and Research (A*STAR), Singapore (grant number SERC 0521010108 'Droplet-based micro/nanofluidics' ). 


\section{References}

[1] Lee S, Choi S U S, Li S and Eastman J A 1999 J. Heat Transfer 121 280-9

[2] Eastman J A, Choi S U S, Li S, Yu W and Thompson L J 2001 Appl. Phys. Lett. 78 718-20

[3] Murshed S M S, Leong K C and Yang C 2005 Int. J. Therm.Sci. 44 367-73

[4] Murshed S M S, Leong K C and Yang C 2006 J. Phys. D: Appl. Phys. 39 5316-22

[5] Li C H and Peterson G P 2006 Appl. Phys. Lett. 99 084314-1-084314-8

[6] Choi S U S, Zhang Z G and Keblinski P 2004 Encyclopedia of Nanoscience and Nanotechnology vol 6, ed H S Nalwa (Los Angeles, CA: American Scientific Publishers) pp 757-73

[7] Nguyen N T, Ting T H, Yap Y F, Wong T N, Chai J C K, Ong W L, Zhou J L, Tan S H and Yobas L 2007 Appl. Phys. Lett. 91 084102-1-084102-3

[8] Tice J D, Lyon A D and Ismagilov R F 2004 Anal. Chim. Acta 507 73-7

[9] Ting T H, Yap Y F, Nguyen N T, Wong T N, Chai J C K and Yobas L 2006 Appl. Phys. Lett. 89 2234101-1-234101-3

[10] Wasan D T and Nikolov A D 2003 Nature 423 156-9

[11] Pak B C and Cho Y I 1998 Exp. Heat Transfer 11 151-70

[12] Prasher R S, Song D, Wang J and Phelan P E 2006 Appl. Phys. Lett. 89 133108-1-133108-3

[13] Murshed S M S, Leong K C and Yang C 2008 Int. J. Therm.Sci. 47 560-8

[14] Joanicot M and Ajdari A 2005 Science 309 887-8

[15] Song H, Chen D L and Ismagilov R F 2006 Angew. Chem. Int. Edn 45 733656

[16] Whitesides G M 2006 Nature 442 368-73

[17] Christopher G F and Anna S L 2007 J. Phys. D: Appl. Phys.40 R319-36

[18] Eastoe J and Dalton J S 2000 Adv. Colloid Interface Sci.85 103-44

[19] Nguyen N T and Wereley S T 2006 Fundamentals and Applications of Microfluidics 2nd edn (London: Artech House)

[20] Girifalco L A and Good R J 1957 J. Phys. Chem. 61 904-9

[21] Einstein A 1956 Investigations on the Theory of the Brownian Movement (New York: Dover)

[22] Frohn A and Roth N 2000 Dynamics of Droplets (Berlin: Springer)

[23] Binks B P 2002 Curr. Opin. Colloid Interface Sci. 7 21-41

[24] Bresme F and Faraudo J 2007 J. Phys.: Condens. Matter 19375110

[25] Lide D R 2007 CRC Handbook of Chemistry and Physics (Boca Raton, FL: Taylor and Francis)

[26] Kampmeyer P M 1952 J. Appl. Phys. 23 99-102

[27] Garstecki P, Fuerstman M J, Stone H A and Whitesides G M 2006 Lap Chip 6 437-46

[28] Xu J H, Li S W, Tan J, Wang Y J and Luo G S 2006 AIChE J.52 3005-10 


\section{List of Figures}

Figure 1. Schematic concept of T-junction microfluidic device.

Figure 2. Comparisons of measured surface tensions of DI water, nanofluid and data obtained from the literature.

Figure 3. Temperature dependence of interfacial tension of DIW/mineral oil and nanofluid /mineral oil.

Figure 4. Comparisons of measured viscosity of DI water and nanofluid with data obtained from the literature.

Figure 5. A typical image of droplet formation process at T-Junction.

Figure 6. Temperature dependence of the droplet size at the T-junction. 


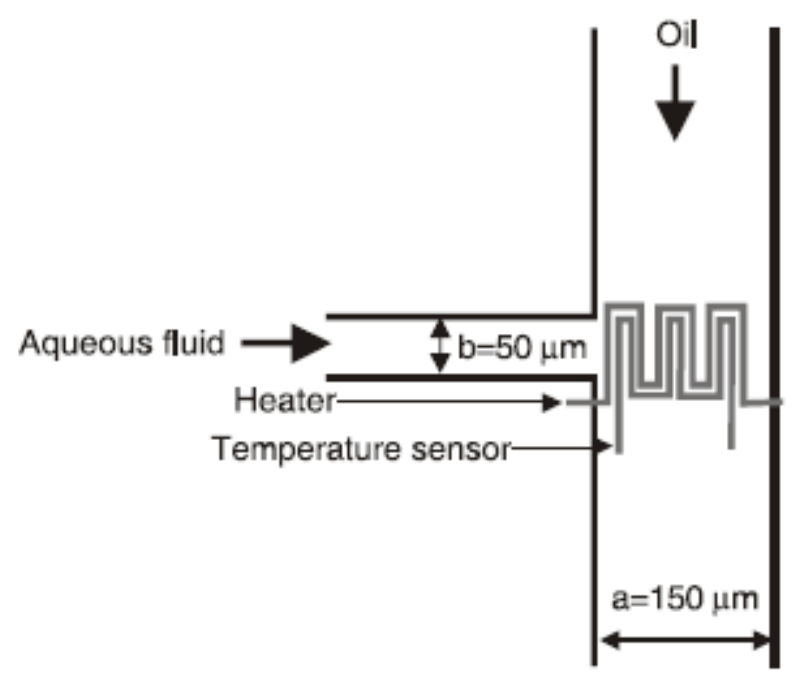

Figure 1 


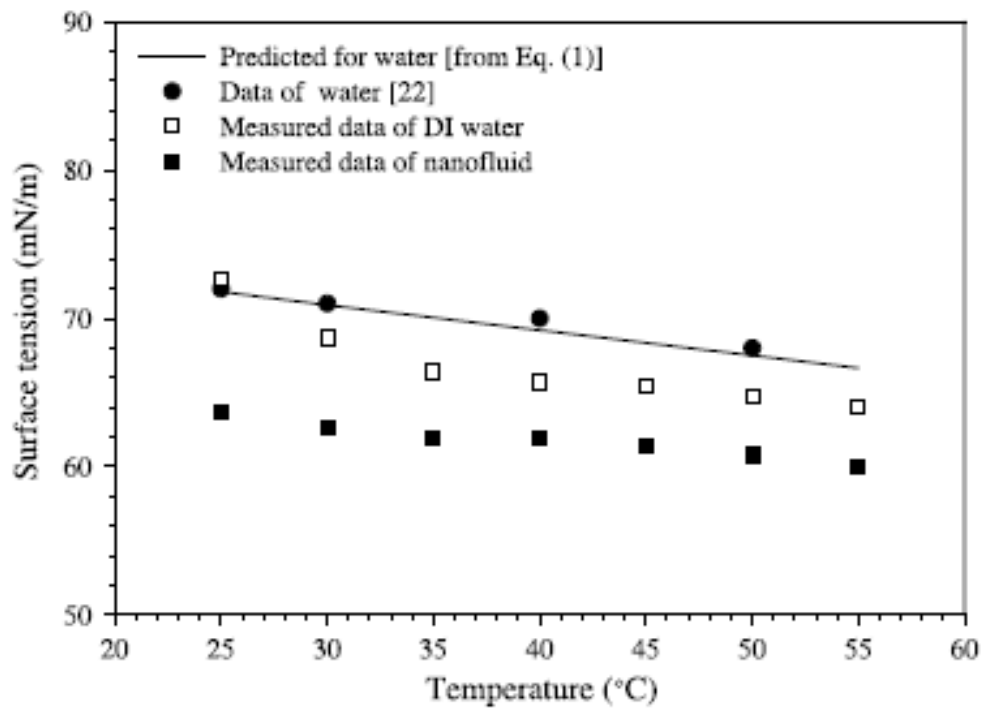

Figure 2 


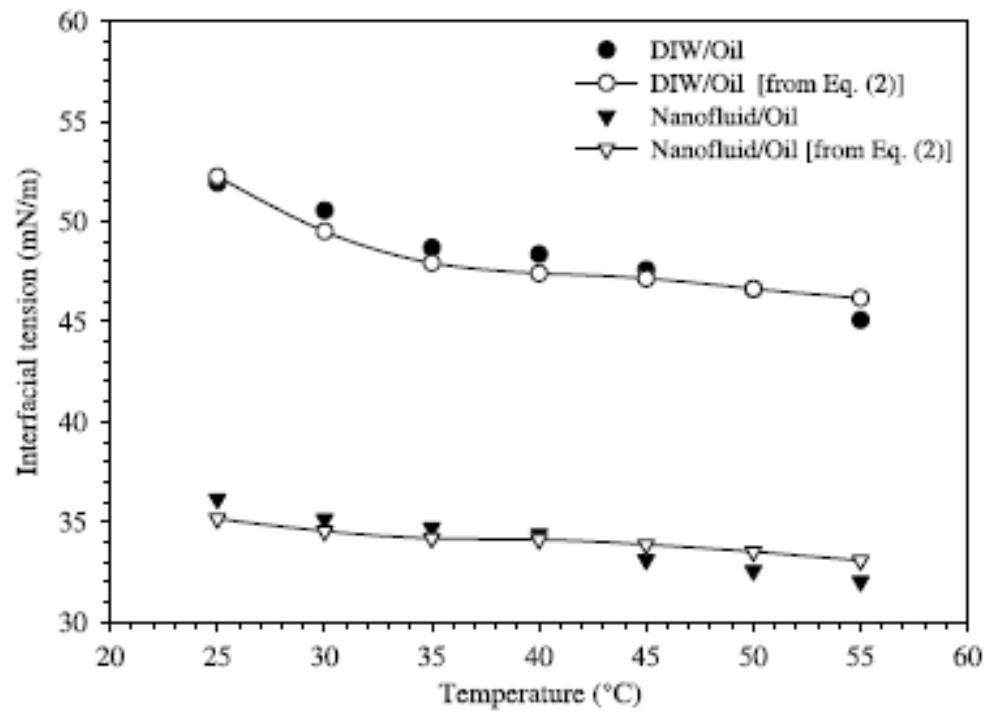

Figure 3 


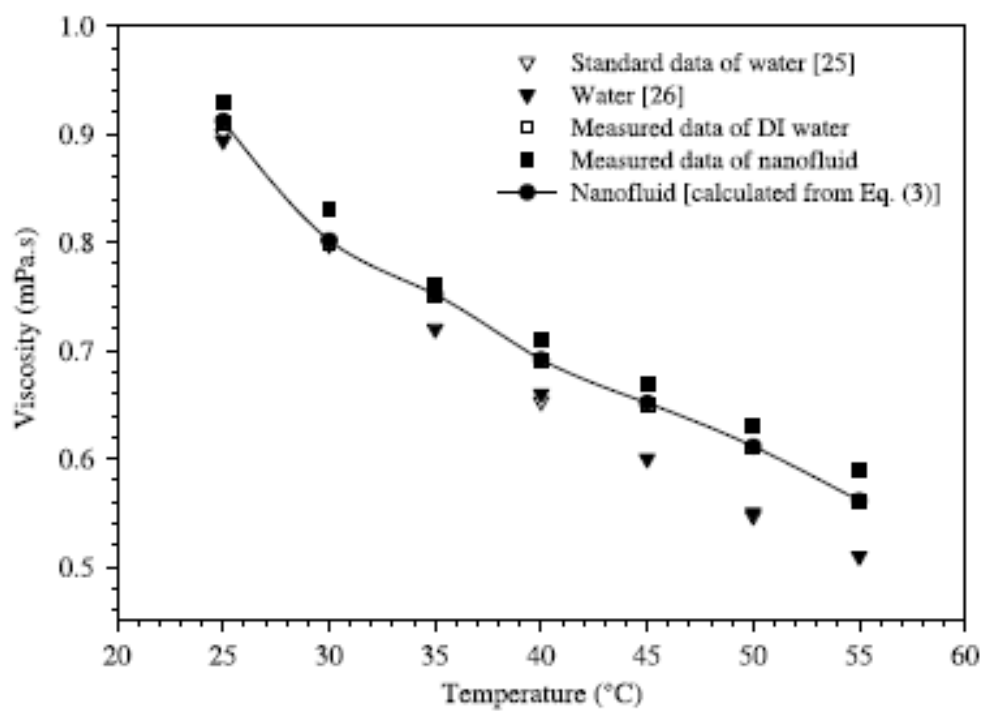

Figure 4 


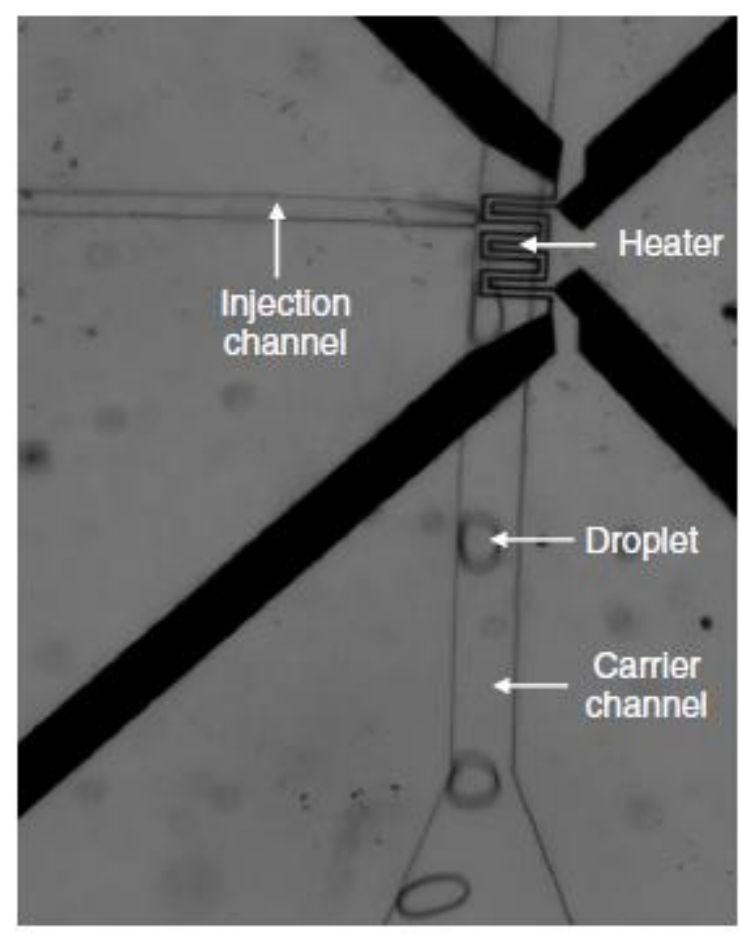

Figure 5 


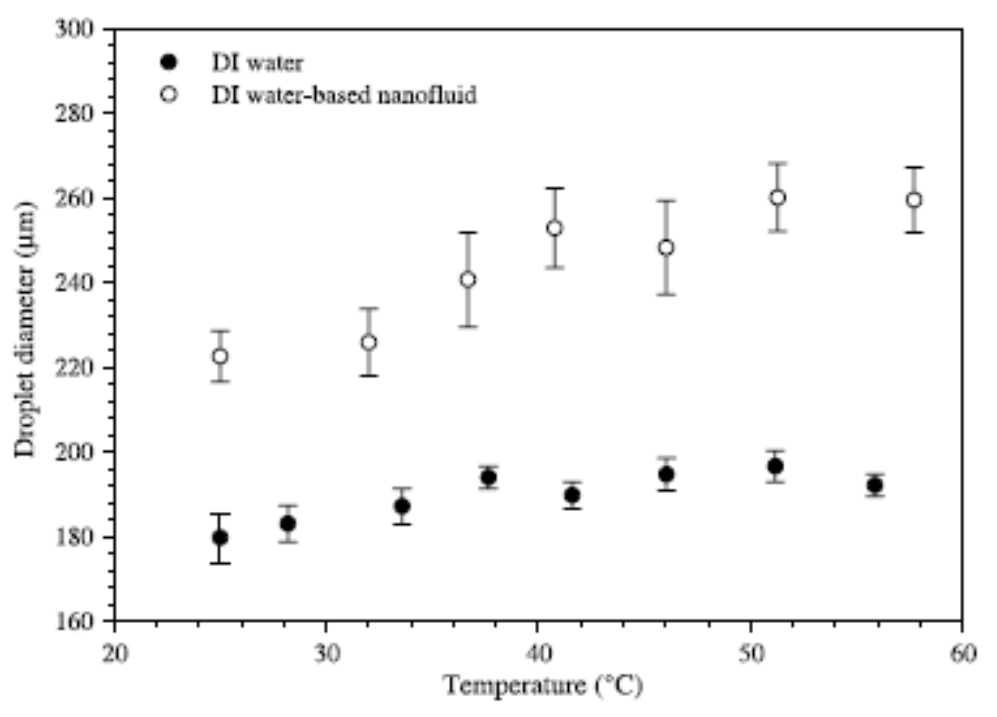

Figure 6 